\title{
Installation of the Ether Bridge of Lolines by the Iron- and 2-Oxoglutarate-Dependent Oxygenase, LolO: Regio- and Stereochemistry of Sequential Hydroxylation and Oxacyclization Reactions
}

\author{
Juan Pan, ${ }^{\perp, \S}$ Minakshi Bhardwaj, ${ }^{\perp, \dagger}$ Bo Zhang, ${ }^{\S}$ Wei-chen Chang, ${ }^{\S}{ }^{\circledR}$ Christopher L. Schardl, ${ }^{*}$, \\ Carsten Krebs, ${ }^{*},{ }^{\circledR}$ Robert B. Grossman, ${ }^{*}{ }^{\dagger} \odot$ and J. Martin Bollinger, Jr. ${ }^{*}, \S$
}

${ }^{\S}$ Department of Chemistry and Department of Biochemistry and Molecular Biology, The Pennsylvania State University, University Park, Pennsylvania 16802, United States

${ }^{\dagger}$ Department of Chemistry and ${ }^{\ddagger}$ Department of Plant Pathology, University of Kentucky, Lexington, Kentucky 40546-0312, United States

\section{Supporting Information}

ABSTRACT: The core of the loline family of insecticidal alkaloids is the bicyclic pyrrolizidine unit with an additional strained ether bridge between carbons 2 and 7. Previously reported genetic and in vivo biochemical analyses showed that the presumptive iron- and 2-oxoglutarate-dependent $(\mathrm{Fe} /$ 2OG) oxygenase, LolO, is required for installation of the

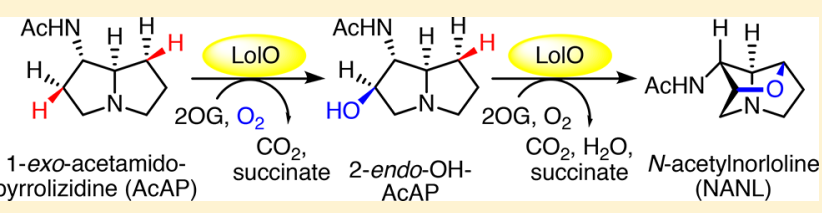

1-exo-acetamido-
pyrrolizidine (AcAP)

ether bridge upon the pathway intermediate, 1-exo-acetamidopyrrolizidine (AcAP). Here we show that LolO is, in fact, solely responsible for this biosynthetic four-electron oxidation. In sequential 2OG- and $\mathrm{O}_{2}$-consuming steps, LolO removes hydrogens from $\mathrm{C} 2$ and $\mathrm{C} 7$ of AcAP to form both carbon-oxygen bonds in $\mathrm{N}$-acetylnorloline (NANL), the precursor to all other lolines. When supplied with substoichiometric 2OG, LolO only hydroxylates AcAP. At higher 2OG:AcAP ratios, the enzyme further processes the alcohol to the tricyclic NANL. Characterization of the alcohol intermediate by mass spectrometry and nuclear magnetic resonance spectroscopy shows that it is 2-endo-hydroxy-1-exo-acetamidopyrrolizidine (2-endo-OH-AcAP). Kinetic and spectroscopic analyses of reactions with site-specifically deuteriated AcAP substrates confirm that the $\mathrm{C} 2-\mathrm{H}$ bond is cleaved first and that the responsible intermediate is, as expected, an $\mathrm{Fe}^{\mathrm{IV}}$-oxo (ferryl) complex. Analyses of the loline products from cultures fed with stereospecifically deuteriated AcAP precursors, proline and aspartic acid, establish that LolO removes the endo hydrogens from $\mathrm{C} 2$ and $\mathrm{C} 7$ and forms both new $\mathrm{C}-\mathrm{O}$ bonds with retention of configuration. These findings delineate the pathway to an important class of natural insecticides and lay the foundation for mechanistic dissection of the chemically challenging oxacyclization reaction.

$\mathrm{L}$ olines are a class of saturated pyrrolizidines with an $\checkmark$ unusual ether bridge and a 1-exo-amino group that is variously substituted to differentiate members of the class (Scheme 1). In cool-season grasses (Poaceae, subfamily Poöideae), lolines are synthesized by endophytic fungi of the genus Epichloë. The fungi are obligate symbionts with the host plant, affording it protection from herbivores by producing several types of objectionable or toxic alkaloid compounds, including lolines. Lolines exhibit biological activities distinct from those of other well-known toxic alkaloids produced by either plants or their fungal symbionts. For example, the plant necine-type pyrrolizidine and fungal ergot alkaloids are both notoriously toxic to mammals such as grazing livestock, ${ }^{1}$ whereas lolines have broad-spectrum antifeedant activity and toxicity against invertebrate herbivores but not mammals. ${ }^{2,3}$ It has been proposed that their complete saturation and uniquely compact tricyclic core, which results from the strained ether bridge, render lolines less susceptible to the mammalian metabolism that converts the other compounds into potent hepatotoxins. ${ }^{4,5}$ These favorable properties could make the breeding of loline-producing grass cultivars a "green" alternative to the use of chemical insecticides. ${ }^{4}$

The biosynthetic pathway to the loline alkaloids has recently been mapped in some detail. ${ }^{3,6-12}$ A cluster of as many as 11 genes (designated $L O L$ ) encodes enzymes that are strictly associated with loline biosynthesis. The first committed step is the condensation of $\mathrm{O}$-acetyl-L-homoserine (derived from Laspartic acid) and L-proline, probably catalyzed by the $\gamma$-type pyridoxal 5'-phosphate(PLP)-dependent enzyme, LolC, to give the diamino diacid, NACPP (Scheme 1). ${ }^{8,11}$ Ensuing cyclization, decarboxylation, and acetylation steps yield 1-exoacetamidopyrrolizidine (AcAP). ${ }^{6}$ Results of in vivo genetic and biochemical studies established that LolO, predicted from its sequence to be an iron- and 2-oxoglutarate-dependent (Fe/ $2 \mathrm{OG}$ ) oxygenase, has an essential role in processing the

Received: February 8, 2018

Revised: March 12, 2018

Published: March 14, 2018 
Scheme 1. Overview of the Loline Alkaloid Biosynthetic Pathway and Representative Lolines ${ }^{a}$

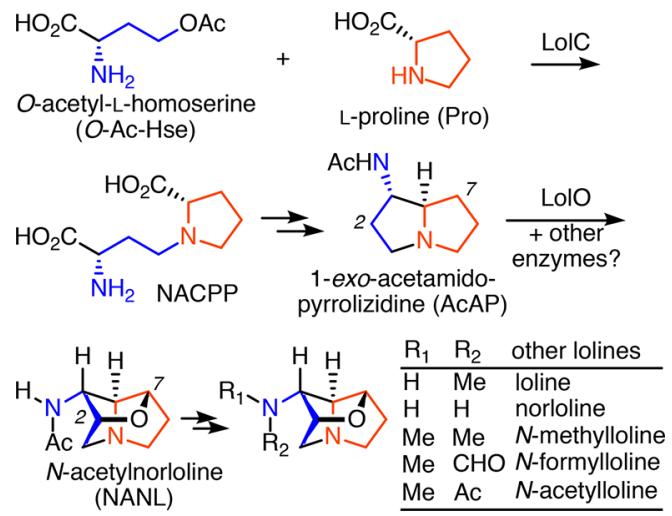

${ }^{a}$ The color coding tracks the atoms of $\mathrm{O}$-acetyl-L-homoserine and Lproline into the loline alkaloids.

pyrrolizidine moiety of AcAP into the tricyclic loline core. Inhibition of lolO expression by RNAi resulted in diminished production of lolines and marked accumulation of AcAP. ${ }^{6}$ Examination of natural fungal variants that produce AcAP as the end product revealed that they all had mutations in the lolO gene. Introduction of a plasmid harboring the wild-type lolO gene into one such mutant strain restored production of $\mathrm{N}$ acetylnorloline (NANL), the expected product of ether-bridge installation upon AcAP. The somewhat surprising implications of these genetic studies-that AcAP, rather than the simpler aminopyrrolizidine, is the entry point to the tricyclic core and NANL the "gateway" loline-were subsequently confirmed by feeding experiments, in which cultures supplied with AcAP deuterium labeled in both the pyrrolizidine core and the $N$ acetyl group produced NANL with both labels in place. Further processing of the exocyclic moiety of NANL by fungal $\mathrm{N}$ acetamidase (LolN), methyltransferase (LolM), and cytochrome P450 (LolP) enzymes, with occasional involvement of a plant acetyltransferase, generates the other known lolines. ${ }^{7,12}$

The observation from these studies that AcAP (but not NANL or another loline) accumulates in the host fungus in the absence of LolO implicated this presumptive Fe/2OG oxygenase in at least initiating, and possibly completing, ether-bridge installation. Published work on other $\mathrm{Fe} / 2 \mathrm{OG}$ enzymes would provide precedent for either possibility. In the conserved mechanistic strategy of the enzyme family, a nonheme $\mathrm{Fe}^{\mathrm{II}}$ cofactor serves to couple reductive activation of $\mathrm{O}_{2}$ with oxidative decarboxylation of $2 \mathrm{OG}^{13,14}$ In each case, an $\mathrm{Fe}^{\mathrm{IV}}$-oxo (ferryl) complex is formed and initiates processing of the substrate. Well-studied transformations of aliphatic carbon centers begin with transfer of a hydrogen atom $\left(\mathrm{H}^{\bullet}\right)$ to the ferryl moiety, producing a carbon-centered radical. In the most common outcome, the radical then couples with the $\mathrm{Fe}^{\mathrm{III}}$ coordinated oxygen, resulting in hydroxylation. Other modes of decay of the substrate radical result in halogenation and desaturation outcomes. Several family members catalyze multiple reaction types in sequence. For example, hyoscyamine $6 \beta$-hydroxylase $(\mathrm{H} 6 \mathrm{H})$ mediates successive hydroxylation and cyclization steps to install the epoxide moiety of the plantderived anesthetic drug, scopolamine, ${ }^{15,16}$ and clavaminate synthase (CAS) mediates hydroxylation and (after an intervening hydrolytic step catalyzed by another enzyme) oxacyclization and desaturation reactions on the pathway to the antibiotic clavulanic acid. ${ }^{17-20}$ Analogously, LolO could effect sequential hydroxylation and oxacyclization steps to install the loline ether bridge. On the other hand, no available data preclude the alternative possibility that LolO merely hydroxylates AcAP and cyclization is then promoted by another enzyme, which, in the aforementioned in vivo studies, would have been present to process the hypothetical LolO product. Indeed, the lolE gene appears also to encode an $\mathrm{Fe} / 2 \mathrm{OG}$ oxygenase, and it has been suggested that the LolE protein product could be responsible for a step in ether-bridge installation. ${ }^{10}$

In this work, we have (i) demonstrated in vitro conversion of AcAP to NANL by LolO, (ii) mapped the sequence of events as hydroxylation of $\mathrm{C} 2$ followed by ring closure upon $\mathrm{C} 7$, and (iii) delineated the stereochemical course of both steps as endo$\mathrm{H}$ abstraction followed by $\mathrm{C}-\mathrm{O}$ bond formation with retention of configuration. By establishing that $\mathrm{LolO}$ is solely responsible for installation of the loline ether bridge, this study provides another example of the switching of regiochemistry and outcome by a member of the versatile $\mathrm{Fe} / 2 \mathrm{OG}$ oxygenase class. In addition, the results establish the necessary foundation for dissection of the mechanism of the oxacyclization step, a reaction type that is among the least well understood within the impressive repertoire of the class.

\section{RESULTS AND DISCUSSION}

LolE Is Dispensible for Ether-Bridge Installation. As a first step toward defining the role of LolO in ether-bridge installation, we explicitly evaluated the published proposal that LolE might share responsibility by catalyzing one of the two required two-electron oxidations. ${ }^{6}$ The absence of a functional lolE gene, which we replaced with a selectable marker gene for hygromycin resistance, did not affect the profile of lolines produced by the host fungus. ${ }^{21}$ This observation rules out an essential role for LolE in ether-bridge installation and leaves the enzyme without any assigned role in the overall biosynthetic pathway. It is likely that this second presumptive $\mathrm{Fe} / 2 \mathrm{OG}$ oxygenase mediates a bifurcating step to other (currently unidentified) metabolites.

Recombinant LolO Catalyzes the Conversion of AcAP to NANL in Vitro. To test the alternative hypothesis that LolO alone is fully competent to convert AcAP to NANL, we sought to reconstitute the reaction in vitro with purified enzyme (expressed heterologously in Escherichia coli) and smallmolecule components. When supplied with $\mathrm{Fe}^{\mathrm{II}},( \pm)$-AcAP, and excess 2OG under an air atmosphere, purified LolO converted AcAP to NANL, with an associated change in the mass-to-charge ratio $[\Delta(\mathrm{m} / z)]$ of +14 (Figure 1A, green and blue traces). This result establishes that LolO alone catalyzes oxidation of both $\mathrm{C} 2$ and $\mathrm{C} 7$ of AcAP to introduce the strained ether bridge. The enzyme can thus promote the final transformation of a five-step chemoenzymatic synthesis of a loline alkaloid from proline (the four chemical steps to AcAP are outlined below); this route is markedly more concise than any previously reported, purely chemical, synthetic procedure. $^{22-28}$

Detection of a Hydroxylated Intermediate Produced from AcAP by LolO. With a few documented exceptions, ${ }^{29,30}$ $\mathrm{Fe} / 2 \mathrm{OG}$ oxygenases balance the four-electron reduction of $\mathrm{O}_{2}$ with two coupled two-electron oxidations, the first fragmenting $2 \mathrm{OG}$ to $\mathrm{CO}_{2}$ and succinate and the second targeting a carbon center in the primary substrate. The conversion of AcAP to NANL is a four-electron oxidation and requires removal of 
A

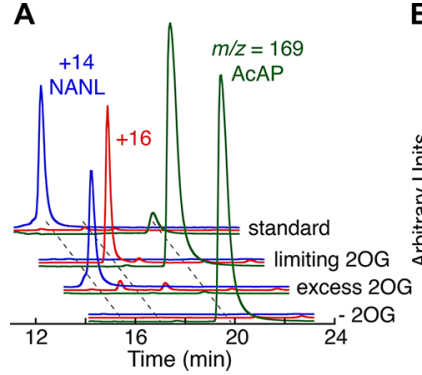

B $[2 \mathrm{OG}] /[(1 S)-\mathrm{AcAP}]$

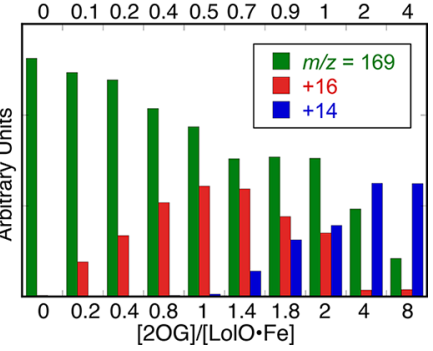

Figure 1. In vitro conversion by LolO of AcAP into NANL via a hydroxylated intermediate. (A) Liquid chromatography-mass spectrometry (LC-MS) analysis of products from incubation of purified LolO with $\mathrm{Fe}^{\mathrm{II}},( \pm)$-AcAP, and varying quantities of the co-substrate, 2OG, under an air atmosphere at room temperature. (B) Variation of the intensities of LC-MS peaks for AcAP (green), the OH-AcAP intermediate (red), and NANL (blue) in analysis of LolO reactions with varying $2 \mathrm{OG}$ levels. Anoxic reaction solutions contained $0.13 \mathrm{mM}$ LolO, $0.10 \mathrm{mM} \mathrm{Fe}$ II, $0.40 \mathrm{mM}( \pm)$-AcAP, and $0-0.80 \mathrm{mM} 2 \mathrm{OG}(0-8$ equiv of LolO. $\mathrm{Fe}^{\mathrm{II}}$ reactant) in $50 \mathrm{mM}$ sodium HEPES buffer $(\mathrm{pH}$ 7.5). Following injection of an equal volume of air-saturated buffer $\left(\sim 0.19 \mathrm{mM} \mathrm{O} \mathrm{O}_{2}\right.$ after mixing), reactions were allowed to proceed at room temperature for $10 \mathrm{~min}$ prior to LC-MS analysis.

hydrogens from two unactivated carbon centers. This conversion is, therefore, expected to require two sequential reactions, each consuming 1 equiv of 2OG. By limiting 2OG in reaction mixtures containing excess $\mathrm{O}_{2}$ and $( \pm)$-AcAP, we resolved the two steps. Liquid chromatography-mass spectrometry (LC-MS) chromatograms from reactions with 2OG:LolO $\cdot \mathrm{Fe}^{\mathrm{II}}$ ratios of $\leq 1$ exhibited a new peak with a $\Delta(m / z)$ of +16 relative to the substrate (Figure 1A, red traces) but no detectable peak at a $\Delta(\mathrm{m} / z)=+14$ for NANL. When we substituted ${ }^{16} \mathrm{O}_{2}$ with ${ }^{18} \mathrm{O}_{2}$ in the same experiment, the $\Delta(\mathrm{m} / z)$ of the new peak increased to +18 relative to the substrate (Figure S1). The observed shift confirmed that the associated compound resulted from hydroxylation of AcAP.

To demonstrate that the hydroxylated compound is an authentic intermediate on the pathway to the NANL product, we analyzed the products of LolO reactions performed with varying quantities of $2 \mathrm{OG}$ (Figure 1B). At 2OG:LolO $\cdot \mathrm{Fe}^{\mathrm{II}}$ ratios of $<1$, the intensity of the $\Delta(\mathrm{m} / z)=+16$ peak increased with increasing [2OG], consistent with the strict dependence of the hydroxylation reaction on the co-substrate. By contrast, once the 2OG:LolO $\cdot \mathrm{Fe}^{\mathrm{II}}$ ratio was increased further in excess of 1 , the intensity of the $\Delta(\mathrm{m} / \mathrm{z})=+16$ peak decreased with increasing $[2 \mathrm{OG}]$, becoming barely detectable by a $2 \mathrm{OG}$ :LolO. $\mathrm{Fe}^{\mathrm{II}}$ ratio of 4 . The diminished yield at high $2 \mathrm{OG}: \mathrm{LolO} \cdot \mathrm{Fe}^{\mathrm{II}}$ ratios is indicative of further processing of the hydroxylated compound in a second 2OG-dependent step. Importantly, in this phase of the titration, the $\Delta(\mathrm{m} / z)=+14$ peak, corresponding to NANL, increased with increasing [2OG], showing that the hydroxylated compound was converted to NANL. The overall conversion catalyzed by LolO can thus be depicted as in Scheme 2. As noted above, similar hydroxylation-oxacyclization sequences have been reported for the related $\mathrm{Fe} / 2 \mathrm{OG}$ oxygenases, $\mathrm{H} 6 \mathrm{H}$ and CAS.

The observation that far more than half of the synthetic, racemic AcAP substrate is consumed at the highest 2OG:LolO. $\mathrm{Fe}^{\mathrm{II}}$ ratios interrogated in the experiments depicted in Figure 1 is surprising, given that most enzymes process only one enantiomer of a chiral substrate. The ability of LolO to process the unnatural AcAP enantiomer (albeit with diminished
Scheme 2. Overall Transformation Catalyzed by LolO

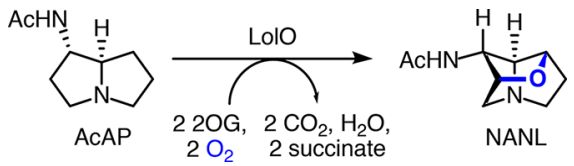

efficiency) hints at an unusual plasticity of its active site. However, this enantiomer is unlikely to be converted to the enantiomer of NANL, as the intensity of the +14 peak did not increase concomitantly with consumption of the second half of the substrate.

Identification of the Hydroxylated Intermediate as 2endo-Hydroxy-1-exo-acetamidopyrrolizidine (2-endoOH-AcAP). To define the structure of the hydroxylated intermediate, we performed the LolO-mediated oxidation of AcAP on a large scale under conditions maximizing the intensity of the $\Delta(\mathrm{m} / z)=+16$ peak, and we purified the associated compound by high-performance liquid chromatography (HPLC). Upon isolation, the compound contained ammonium acetate (from the mobile phase) and at least one unidentified minor contaminant, but it was sufficiently pure to permit identification from its ${ }^{1} \mathrm{H}$ nuclear magnetic resonance (NMR), ${ }^{1} \mathrm{H}-{ }^{1} \mathrm{H}$ COSY, and nuclear Overhauser effect (NOE) spectra (Figures $\mathrm{S} 2-\mathrm{S} 5$ ). The ${ }^{1} \mathrm{H}$ NMR spectrum exhibited two doublets of doublets at $\delta 3.25(\mathrm{~J}=12.6$ and $6.6 \mathrm{~Hz})$ and $3.90(\mathrm{~J}$ $=12.7$ and $5.7 \mathrm{~Hz}$ ), which can be attributed to the geminal pair of $\mathrm{H}$ atoms at $\mathrm{C} 3$ (Figure 2); in the COSY spectrum, these two

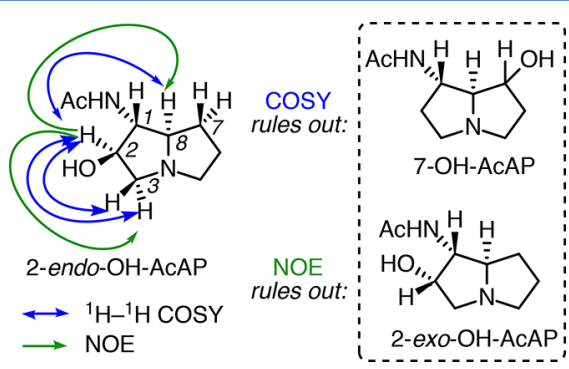

Figure 2. Selected ${ }^{1} \mathrm{H}-{ }^{1} \mathrm{H}$ COSY and NOE correlations and assignment of the structure of 2-endo-OH-AcAP.

resonances correlated only to one another and to the most downfield resonance, a quartet at $\delta 4.43$, assigned to the $\mathrm{H}$ atom attached to $\mathrm{C} 2$. In the COSY spectrum, the quartet at $\delta$ 4.43 also correlated to a triplet at $\delta 4.16$, assigned to the $\mathrm{H}$ atom at $\mathrm{C} 1$. These data are consistent with the presence of the hydroxyl group on $\mathrm{C} 2$ but not with its presence on C7. Furthermore, irradiation of the resonance attributed to the $\mathrm{H}$ atom attached to $\mathrm{C} 2$ (quartet at $\delta 4.43$ ) caused strong NOE enhancements of two resonances, one assigned to the hydrogen attached to $\mathrm{C} 8$ (quartet at $\delta 4.08$ ) and one attributed to one of the two hydrogens attached to C3 (dd at $\delta 3.90$ ), but only a very small NOE enhancement of the resonance attributed to the hydrogen at $\mathrm{C} 1$ (triplet at $\delta 4.16$ ). These data are uniquely consistent with installation of the $\mathrm{C} 2$ hydroxyl group with endo stereochemistry, thus identifying the intermediate as 2-endoOH-AcAP.

Order of $\mathrm{C}-\mathrm{H}$ Cleavage Steps Determined by Transient Kinetic/Spectroscopic Experiments with Deuterium-Labeled Substrates. Previous studies of $\mathrm{Fe} / 2 \mathrm{OG}$ hydroxylases, halogenases, and the epimerase/desaturase, CarC, demonstrated accumulation of the $\mathrm{C}-\mathrm{H}$ bond-cleaving ferryl 
complexes and substrate deuterium kinetic isotope effects $\left({ }^{2} \mathrm{H}\right.$ KIE) of $20-60$ on their decay by $\mathrm{H}^{\bullet}$ abstraction. ${ }^{14,31,32}$ To verify that $\mathrm{C} 2$, rather than $\mathrm{C}$, is hydroxylated in the first step of ether-bridge installation by LolO, we synthesized sitespecifically deuterium-labeled AcAPs and tested the prediction that the reaction with the C2-labeled substrate would exhibit a large ${ }^{2} \mathrm{H}$-KIE upon decay of the ferryl intermediate, but the reaction of the C7-labeled substrate would not.

Synthesis of $( \pm)-7,7-\left[{ }^{2} \mathrm{H}_{2}\right]$ ACAP. Installation of deuterium atoms at $\mathrm{C} 7$ of AcAP required $3,3-\left[{ }^{2} \mathrm{H}_{2}\right]$ Pro. The literature route to it was unsatisfactory, ${ }^{36}$ and so we prepared it in racemic form by reducing 3-keto proline derivative $1^{37}$ with $\mathrm{NaBD}_{4}$, tosylating the resulting alcohol, reducing the resulting tosylate with $\mathrm{NaBD}_{4}$, and deprotecting with $6 \mathrm{M} \mathrm{HCl}$ (Scheme 3 ). Using procedures that we previously reported, ${ }^{9}$ we then

Scheme 3. Synthesis of $( \pm)-7,7-\left[{ }^{2} \mathbf{H}_{2}\right]$ AcAP

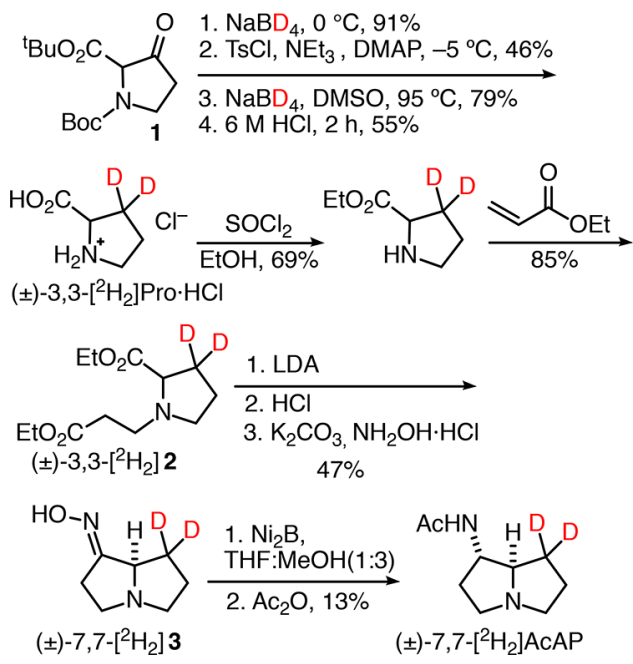

esterified ( \pm )-3,3- $\left[{ }^{2} \mathrm{H}_{2}\right]$ Pro and allowed the resulting ester to undergo conjugate addition to ethyl acrylate to give $( \pm)-3,3-$ $\left[{ }^{2} \mathrm{H}_{2}\right] 2$. We then subjected this diester to Dieckmann condensation, hydrolysis, decarboxylation, and $\mathrm{NH}_{2} \mathrm{OH} \cdot \mathrm{HCl}$ condensation to give oxime $( \pm)-7,7-\left[{ }^{2} \mathrm{H}_{2}\right] 3$. Previously, we had reduced $( \pm)-3,3-\left[{ }^{2} \mathrm{H}_{2}\right] 3$ with Raney nickel and then trideuteroacetylated the nascent amine in an attempt to make ( \pm - $-2^{\prime}, 2^{\prime}, 2^{\prime}, 3,3-\left[{ }^{2} \mathrm{H}_{5}\right]$ AcAP. ${ }^{7}$ This step gave a modest yield and also caused a considerable loss of deuterium, resulting in a product with either one $(85 \%)$ or no $(13 \%)$ deuterium atoms. Loss of deuterium was even more pronounced in the attempted reduction of $( \pm)-7,7-\left[{ }^{2} \mathrm{H}_{2}\right] 3$. After much experimentation with alternative reducing agents, which either gave unacceptably poor yields or favored the undesired diastereomer, we followed a precedent from ref 38 by treating $( \pm)-7,7-\left[{ }^{2} \mathrm{H}_{2}\right] 3$ with nickel boride generated in situ from $\mathrm{NiCl}_{2}$ and $\mathrm{NaBH}_{4}$. Subsequent acetylation gave $( \pm)-7,7-\left[{ }^{2} \mathrm{H}_{2}\right] \mathrm{AcAP}$ in a yield $(13 \%)$ that was better than that of the Raney $\mathrm{Ni}$ reaction and without a detectable loss of deuterium [ $97 \% d_{2}$ by high-resolution mass spectrometry (HRMS)].

Synthesis of $( \pm)-2,2,8-\left[{ }^{2} \mathrm{H}_{3}\right]$ AcAP. To install deuterium atoms at $\mathrm{C} 2$, we subjected diester $\mathbf{2}^{11}$ to Dieckmann condensation and then used $\mathrm{DCl}$ in $\mathrm{D}_{2} \mathrm{O}$ to effect hydrolysis, decarboxylation, and deuterium incorporation (Scheme 4). Formation of oxime 2,2,8- $\left[{ }^{2} \mathrm{H}_{3}\right] 3$ and nickel boride reduction proceeded without any loss of deuterium, and subsequent acetylation gave $( \pm)-2,2,8-\left[{ }^{2} \mathrm{H}_{3}\right] \mathrm{AcAP}\left(88 \% d_{3}\right.$ by HRMS). We
Scheme 4. Synthesis of $( \pm)-2,2,8-\left[{ }^{2} \mathrm{H}_{3}\right] \mathrm{AcAP}$

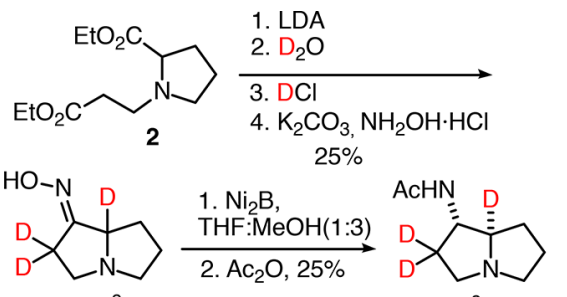

$( \pm)-2,2,8-\left[{ }^{2} \mathrm{H}_{3}\right] 3$

$( \pm)-2,2,8-\left[{ }^{2} \mathrm{H}_{3}\right] \mathrm{AcAP}$

did not view the incorporation of the additional deuterium at C8 as a detriment, because our prior demonstration of the retention of this hydron in the biosynthetic conversion of AcAP to $\mathrm{NANL}^{6}$ implied that it would not impact kinetic analysis of the LolO reaction. Moreover, the $\mathrm{C} 8-\mathrm{H} / \mathrm{D}$ exchange demonstrated here validates our suppositions in prior studies that $\mathrm{C} 2$ of the proline starting material was racemized and the AcAP derived therefrom was thus racemic.

Kinetic and Spectroscopic Evidence for C2 Hydroxylation by a Ferryl Intermediate. The conserved experimental hallmarks of the ferryl complexes in Fe/2OG oxygenases are their transient ultraviolet absorption at $\sim 320$ $\mathrm{nm}$ and Mössbauer quadrupole doublet spectra with isomer shift parameters, $\delta$, of $0.2-0.3 \mathrm{~mm} / \mathrm{s}$ and quadrupole splitting parameters, $\Delta E_{\mathrm{Q}}$, of approximately $-1 \mathrm{~mm} / \mathrm{s}^{14,32-35}$ With the required deuterium-labeled substrates in hand, we tested for both accumulation of the ferryl complex and a ${ }^{2} \mathrm{H}-\mathrm{KIE}$ on its decay by stopped-flow absorption and freeze-quench Mössbauer spectroscopies, as we have previously done for other $\mathrm{Fe} /$ $2 \mathrm{OG}$ oxygenases. $^{29,34,35,39,40}$ Mixing an $\mathrm{O}_{2}$-free solution containing LolO, $\mathrm{Fe}^{\mathrm{II}}, 2 \mathrm{OG}$, and $( \pm)$-AcAP with air-saturated buffer at $5{ }^{\circ} \mathrm{C}$ resulted in transient absorption at $320 \mathrm{~nm}$ (Figure 3A, red trace), and the presence of the characteristic quadrupole doublet features in the $4.2 \mathrm{~K}, 53 \mathrm{mT}$ Mössbauer spectra of samples freeze-quenched at short reaction times (Figure 3B, spectra i-iii) confirmed that the transient ultraviolet absorption arose from the ferryl complex. The kinetics of the intermediate were hardly altered when we used $7,7-\left[{ }^{2} \mathrm{H}_{2}\right]$ AcAP in place of the unlabeled substrate (in Figure $3 \mathrm{~A}$, compare red and blue traces), but the use of 2,2,8$\left[{ }^{2} \mathrm{H}_{3}\right]$ AcAP resulted in enhanced accumulation and slower decay of the transient ultraviolet (UV) absorption (Figure 3A, green trace) and Mössbauer quadrupole doublet features associated with the ferryl complex (Figure 3B, spectra iv and $\mathrm{v})$. The marked stabilization of the ferryl complex by deuterium at C2, and the absence of a significant effect of deuterium at C7, confirm that the first step in ether-bridge installation by LolO is transfer of $\mathrm{H}^{\bullet}$ from $\mathrm{C} 2$ of AcAP to initiate hydroxylation of that position.

In the freeze-quench Mössbauer experiment with 2,2,8$\left[{ }^{2} \mathrm{H}_{3}\right] \mathrm{AcAP}$, the ferryl complex was observed to accumulate to a maximum of only $\sim 29 \%$ of the total iron in the sample, less than half the quantity predicted on the basis of its relatively fast formation and much slower decay (Table 1). This substoichiometric accumulation arose from the use of slightly less than saturating substrate in that experiment [ 1 equiv of the correct enantiomer, giving $\sim 92 \%$ saturation (see Figure S6)] and, more importantly, from the fact that only $0.4 \pm 0.1$ of the $\mathrm{LolO} \cdot \mathrm{Fe}^{\mathrm{II}} \cdot 2 \mathrm{OG} \cdot \mathrm{AcAP}$ complex is competent to react rapidly with $\mathrm{O}_{2}$ to generate the intermediate. Such fractional reactivity has been encountered for every other Fe/2OG enzyme that has 


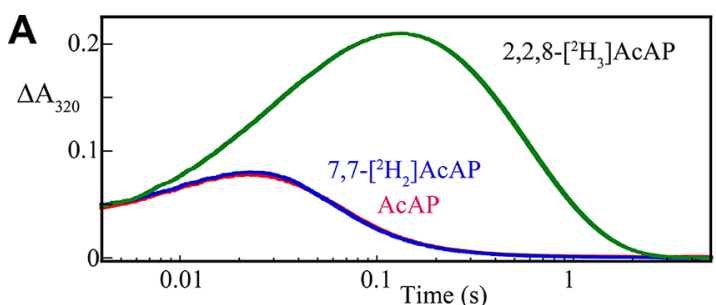

B

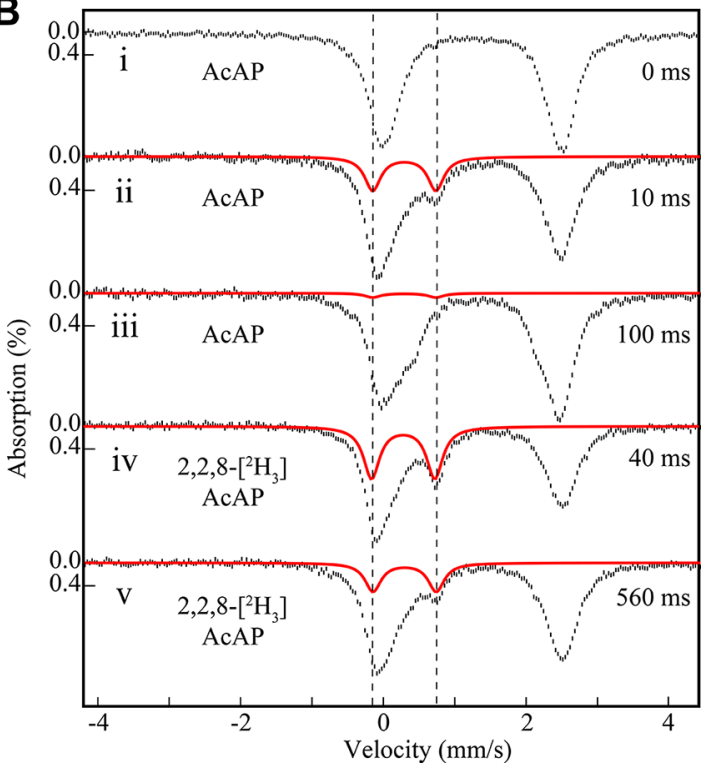

Figure 3. Kinetic and spectroscopic evidence for abstraction of hydrogen from $\mathrm{C} 2$ of AcAP by a ferryl intermediate. (A) Change in absorbance at $320 \mathrm{~nm}$ following mixing at $5{ }^{\circ} \mathrm{C}$ of an anoxic solution containing $1.1 \mathrm{mM}$ LolO, $0.80 \mathrm{mM} \mathrm{Fe}^{\mathrm{II}}, 5.0 \mathrm{mM} 2 \mathrm{OG}$, and the indicated $( \pm)$-AcAP isotopolog $(4.0 \mathrm{mM})$ in $50 \mathrm{mM}$ sodium HEPES ( $\mathrm{pH} 7.5)$ with an equal volume of air-saturated buffer $\left(\sim 0.19 \mathrm{mM} \mathrm{O}_{2}\right.$ after mixing). Kinetic constants are listed in Table 1. (B) The $4.2 \mathrm{~K}, 53$ $\mathrm{mT}$ Mössbauer spectra of samples freeze-quenched at the indicated reaction times after mixing of an anoxic solution containing $2.3 \mathrm{mM}$ LolO, $1.8 \mathrm{mM}{ }^{57} \mathrm{Fe}^{\mathrm{II}}, 12 \mathrm{mM} \mathrm{2OG}$, and the indicated ( \pm )-AcAP isotopolog $(3.6 \mathrm{mM})$ in $50 \mathrm{mM}$ sodium HEPES ( $\mathrm{pH} 7.5)$ with an equal volume of $\mathrm{O}_{2}$-saturated buffer $\left(\sim 0.90 \mathrm{mM} \mathrm{O} \mathrm{O}_{2}\right.$ after mixing). Black vertical bars represent experimental data. The red lines are simulations of the quadrupole doublet of ferryl species with the parameters of isomer shift $(\delta=0.28 \mathrm{~mm} / \mathrm{s})$ and quadrupole splitting parameter $\left(\left|\Delta E_{\mathrm{Q}}\right|=0.89 \mathrm{~mm} / \mathrm{s}\right)$ accounting for $16,3,28$, and $15 \%$ of total iron in spectra ii-v, respectively.

Table 1. Observed Kinetic Constants for Formation and Decay of the Ferryl Intermediate during Hydroxylation of C2 of the ( \pm )-AcAP Isotopologs by LolO

\begin{tabular}{lccc}
\multicolumn{1}{c}{ substrate } & $k_{\text {formation }}\left(10^{5} \mathrm{M}^{-1} \mathrm{~s}^{-1}\right)$ & $k_{\text {decay }}\left(\mathrm{s}^{-1}\right)$ & $k_{\mathrm{H}} / k_{\mathrm{D}}$ \\
AcAP & $1.3 \pm 0.2$ & $48 \pm 4$ & - \\
$7,7-\left[{ }^{2} \mathrm{H}_{2}\right]$ AcAP & $1.3 \pm 0.2$ & $47 \pm 5$ & $1.0 \pm 0.2$ \\
$2,2,8-\left[{ }^{2} \mathrm{H}_{3}\right]$ AcAP & $1.3 \pm 0.2$ & $2.0 \pm 0.1$ & $23 \pm 5$ \\
\hline
\end{tabular}

been subjected to this level of transient-state kinetic analysis. $^{29,31,34,41}$

By global simulation of the kinetic data for the reactions with the three substrate isotopologs under varying experimental conditions (e.g., limiting $\mathrm{O}_{2}$, limiting 2OG, etc.), we obtained values for the rate constants for formation and decay of the ferryl complex and observed ${ }^{2} \mathrm{H}-\mathrm{KIE}$ on the $\mathrm{C} 2-\mathrm{H}$ cleavage step (Table 1). The intrinsic ${ }^{2} \mathrm{H}$-KIE for this step may be greater than the observed value of $23 \pm 5$, if pathways not involving $\mathrm{C} 2-\mathrm{H}$ cleavage contribute significantly to ferryl decay when the intermediate is challenged by the heavy hydron at $\mathrm{C} 2$. With the parameters listed in Table 1, both the quantities of the ferryl complex determined in the freeze-quench Mössbauer experiments and the kinetic traces from stopped-flow experiments performed under identical reaction conditions could be accounted for (Figure S7), assuming a reactive fraction of 0.4 and a molar absorptivity of the ferryl complex of $2000 \mathrm{M}^{-1}$ $\mathrm{cm}^{-1}$. The latter value is similar to (albeit somewhat larger than) those determined previously for ferryl complexes in other $\mathrm{Fe} / 2 \mathrm{OG}$ oxygenases.

Determination of the Stereochemical Courses of the Steps in Ether-Bridge Installation by LolO. In formation of the ether bridge, $\mathrm{C}-\mathrm{H}$ bonds to $\mathrm{C} 2$ and $\mathrm{C} 7$ of AcAP are cleaved and a new $\mathrm{C}-\mathrm{O}$ bond to each carbon is formed. The structure of the NANL product implies that the ether oxygen is added to AcAP at the positions initially occupied by the endo hydrogens, but the free-radical nature of this chemistry makes either of two cases possible for each step: the endo hydrogen could be abstracted and the $\mathrm{C}-\mathrm{O}$ bond formed with retention, or the exo hydrogen could be removed and the $\mathrm{C}-\mathrm{O}$ bond formed with inversion. Although the oxygen-rebound mechanism for hydroxylation makes retention of configuration at C2 far more likely than inversion, the situation is less clear for $\mathrm{C} 7$, because the mechanism of ring closure is less well understood. The simplest possibility would involve coordination of the newly installed $\mathrm{C} 2$ oxygen to the $\mathrm{Fe}^{\mathrm{II}}$ cofactor in the reactant complex for the oxacyclization step, followed later by radicaloid coupling of the ferryl-generated substrate radical with the alkoxo, rather than the hydroxo, ligand. This mechanism would be analogous to that proposed for thiazolidine ring closure in the reaction of isopenicillin $N$-synthase (IPNS). ${ }^{42,43}$ However, it is unclear for the case of LolO how the cofactor, which is expected to have one carboxylate and two histidine ligands from the protein as well as a carboxylate and carbonyl oxygen from 2OG (Figure S8), could coordinate the $\mathrm{C} 2-\mathrm{O}$ bond and still activate $\mathrm{O}_{2}$. A second possibility would involve formation of a carbocation by the transfer of an electron from the carbon radical to the $\mathrm{Fe}(\mathrm{III})-\mathrm{OH}$ cofactor state and subsequent polar ring closure by attack of the substrate oxygen. Indeed, this mechanism was recently proposed for closure of the epoxide ring in the antibiotic fosfomycin by $(S)$-2-hydroxypropylphosphonate epoxidase (HppE). ${ }^{44,45}$ Among the evidence cited for this mechanism was the inversion of configuration at $\mathrm{Cl}$ during ring closure. This example shows that the stereochemistry of $\mathrm{H}^{\bullet}$ abstraction and $\mathrm{C}-\mathrm{O}$ coupling is a crucial mechanistic issue in the oxacyclization reactions. To determine the stereochemical courses of both steps in installation of the ether bridge by LolO, we fed stereoselectively deuteriumlabeled precursors, Pro and Asp, to loline-producing cultures and tested for the presence of deuterium in the products.

Stereochemistry of $\mathbf{C 7}-\mathrm{H}$ Cleavage. Previous work showed that $\mathrm{C} 7$ of $\mathrm{N}$-formylloline (NFL) is derived from $\mathrm{C} 3$ of Pro (Scheme 5, left side). ${ }^{8}$ With the reasonable assumption that the $\mathrm{C} 3$ configuration of Pro should remain unchanged until the $\mathrm{C}-\mathrm{H}$ abstraction event, we expected that feeding trans-3$\left[{ }^{2} \mathrm{H}\right]$ Pro would lead to the intermediate 7 -endo- $\left[{ }^{2} \mathrm{H}\right] \mathrm{AcAP}$, and cis-3- $\left[{ }^{2} \mathrm{H}\right]$ Pro to 7-exo- $\left[{ }^{2} \mathrm{H}\right] \mathrm{AcAP}$. Therefore, if LolO removed the endo hydrogen from $\mathrm{C} 7$ of AcAP (blue highlight), then trans-3- $\left[{ }^{2} \mathrm{H}\right]$ Pro would give NFL lacking deuterium at $\mathrm{C}$, and cis-3- $\left[{ }^{2} \mathrm{H}\right]$ Pro would give $7-\left[{ }^{2} \mathrm{H}\right] \mathrm{NFL}$ (blue arrows). By contrast, if LolO abstracted the exo $\mathrm{H}$ atom at $\mathrm{C} 7$ of AcAP (green highlight), then trans-3-[ $\left[{ }^{2} \mathrm{H}\right]$ Pro would give $7-\left[{ }^{2} \mathrm{H}\right] \mathrm{NFL}$, 
Scheme 5. Predicted Hydrogen Isotopic Composition of NFL Obtained from Cultures Fed Stereoselectively Deuterium-Labeled Precursors, Pro and Asp ${ }^{a}$

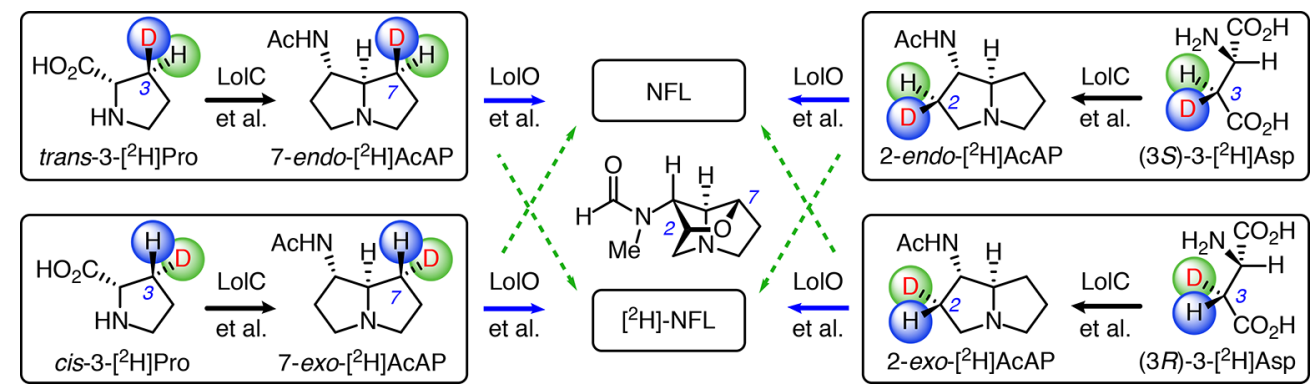

${ }^{a}$ Green and blue spheres depict the relevant hydrons and the positions that they come to occupy in AcAP. The solid blue arrows depict the results of abstraction of the endo hydrons from $\mathrm{C} 2$ and $\mathrm{C} 7$ of AcAP by LolO, and the dashed green arrows depict the results of removal of the exo hydrons.

and cis-3- $\left[{ }^{2} \mathrm{H}\right]$ Pro would give NFL lacking deuterium (green dashed arrows).

Syntheses of cis- and trans-3-[2H]Pro. We began the syntheses of regio- and stereoselectively deuteriated prolines ${ }^{36}$ by protecting commercially available trans-3-hydroxy-L-proline with Boc and tert-butyl groups to give 4 (Scheme 6). ${ }^{11} \mathrm{We}$

Scheme 6. Syntheses of cis- and trans- $3-\left[{ }^{2} \mathrm{H}\right] \mathrm{Pro} \cdot \mathrm{HCl}$

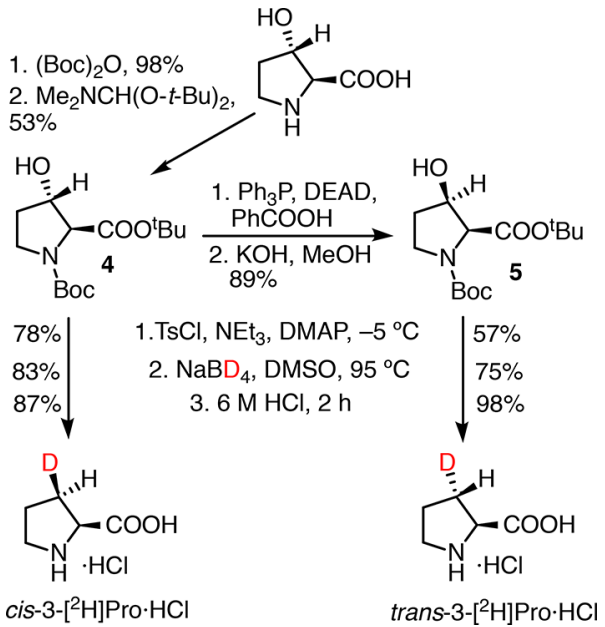

divided 4 into two portions, allowing one portion to undergo a Mitsunobu reaction to give 5 . We then subjected both 5 and the remaining 4 to tosylation, reduction with $\mathrm{NaBD}_{4}$, and deprotection with $6 \mathrm{~N} \mathrm{HCl}$ to give cis- and trans-3- $\left[{ }^{2} \mathrm{H}\right]$ Pro. $\mathrm{HCl}$, respectively.

Culture Feeding and Analysis of Products. We fed loline-producing cultures of Epichloë uncinata e167 with the two Pro isotopologs and measured the deuterium content of the resultant NFL by GC-MS (Figure 4). The spectrum of NFL lacking deuterium had a prominent $\mathrm{m} / z=183$ peak, previously assigned to the $[\mathrm{M}+\mathrm{H}]$ ion, ${ }^{3,8,46}$ and a small $\mathrm{m} / z=184$ peak from species with one atom of a heavier isotope of $\mathrm{C}, \mathrm{N}$, or $\mathrm{O}$ (Figure 4A). The spectrum of NFL from the culture with cis-3$\left[{ }^{2} \mathrm{H}\right]$ Pro displayed a significant enhancement of the $184(+1)$ peak $(30.5 \pm 2.3 \%)$ (Figure 4B), consistent with the incorporation of ${ }^{2} \mathrm{H}$ into much of the NFL, whereas the spectrum of NFL from the culture with trans-3- $\left[{ }^{2} \mathrm{H}\right]$ Pro had a barely detectable peak $(2.9 \pm 0.3 \%)$ at this $m / z$ value (Figure 4C). The small but significant enrichment of ${ }^{2} \mathrm{H}$ from the feeding of trans-3-[ $\left[{ }^{2} \mathrm{H}\right]$ Pro likely arose from contamination of the primary isomer with a small amount of cis-3- $\left[{ }^{2} \mathrm{H}\right]$ Pro, as

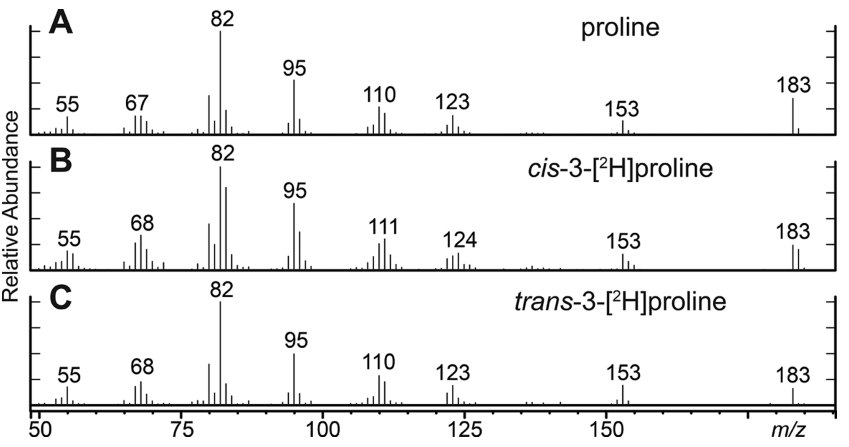

Figure 4. Mass spectra of NFL from loline-producing cultures fed with (A) natural-abundance Pro, (B) cis-3- $\left[{ }^{2} \mathrm{H}\right]$ Pro, and (C) trans-3$\left[{ }^{2} \mathrm{H}\right]$ Pro.

shown in the ${ }^{1} \mathrm{H}$ NMR spectrum of the synthetic material (Figure S9). The incorporation of one deuterium atom into NFL produced from cis-3- $\left[{ }^{2} \mathrm{H}\right]$ Pro implies that LolO abstracts the endo hydrogen from C7 of AcAP (Scheme 5).

Stereochemistry of $\mathrm{C} 2-\mathrm{H}$ Cleavage. Previous work showed that C2 of NFL is derived from C3 of Asp (Scheme 5 , right side) via homoserine. ${ }^{8}$ As analyzed in detail in the Supporting Information, the sequence of biosynthetic steps converting Asp to AcAP (in particular the step attributed to the PLP-dependent enzyme, LolC) is expected to exchange the C2 hydron and one of two diastereotopic $\mathrm{C} 3$ hydrons with solvent (Scheme S1) but not to exchange the other C3 hydron nor to invert or racemize $\mathrm{C} 3$. We thus anticipated that $(1)$ in principle, (3S)-3-[ $\left[{ }^{2} \mathrm{H}\right]$ Asp would yield 2-endo- $\left[{ }^{2} \mathrm{H}\right] \mathrm{AcAP}$ and (3R)-3$\left[{ }^{2} \mathrm{H}\right]$ Asp 2-exo- $\left[{ }^{2} \mathrm{H}\right]$ AcAP but $(2)$ in actuality, one of the two diastereomers would yield AcAP lacking deuterium (Scheme S2). Therefore, if LolO were to remove the endo hydron from C2 of AcAP (blue highlight), then feeding cultures with (3S)-3$\left[{ }^{2} \mathrm{H}\right]$ Asp would necessarily yield NFL lacking deuterium, whereas feeding with (3R)-3-[2 $\mathrm{H}]$ Asp could yield 2-[ $\left.{ }^{2} \mathrm{H}\right] \mathrm{NFL}$ (blue arrows), provided that the other biosynthetic enzymes did not cause exchange of the deuteron with solvent during production of AcAP. Conversely, were LolO to abstract the exo hydron (green highlight), only for (3S)-3-[2 $\mathrm{H}]$ Asp could deuterium possibly be retained in the NFL product of the LolO reaction (green dashed arrows).

We prepared (3S)-2,3- $\left[{ }^{2} \mathrm{H}_{2}\right]$ Asp $\left(89 \% d_{2}\right)$ and (3R)-3$\left[{ }^{2} \mathrm{H}\right]$ Asp $\left(95 \% d_{1}\right)$ chemoenzymatically by literature procedures, except that we used aspartase $\mathrm{B}^{47}$ instead of aspartase $\mathrm{A}^{48,49}$ or 3-methylaspartase ${ }^{50}$ (Scheme 7). As noted in the analysis in the Supporting Information, we expected that the 
C2 hydron of the former compound would be lost to solvent in conversion to AcAP, thus obviating complications from the extraneous deuteron. The two Asp isotopologs were applied to loline-producing cultures, and the isotopic composition of the resultant NFL was determined by GC-MS (Table 2). In the feeding experiments with (3R)-3- $\left[{ }^{2} \mathrm{H}\right]$ Asp, the NFL product was significantly enriched in a species with $m / z+1$ relative to the product from the natural-abundance control, implying the retention of a single deuteron in a significant fraction of the product. By contrast, experiments with (3S)-2,3- $\left[{ }^{2} \mathrm{H}_{2}\right]$ Asp gave no more $\left[{ }^{2} \mathrm{H}\right] \mathrm{NFL}$ (nor $\left[{ }^{2} \mathrm{H}_{2}\right] \mathrm{NFL}$ ) than did the control. These results are most consistent with abstraction of the endo hydrogen from $\mathrm{C} 2$ of AcAP (Table S1). In addition, the absence of any residual deuterium in the NFL formed from the (3S)-2,3- $\left[{ }^{2} \mathrm{H}_{2}\right]$ Asp precursor confirms the expected exchange of the $\mathrm{C} 2$ hydron during the steps leading to AcAP (Scheme S1). Hence, as for the oxidation of C7, LolO also abstracts the endo hydrogen from $\mathrm{C} 2$ and forms the $\mathrm{C} 2-\mathrm{O}$ bond with retention.

Scheme 7. Syntheses of (3R)-3-[ $\left[{ }^{2} \mathrm{H}\right]$ - and (3S)-2,3- $\left[{ }^{2} \mathrm{H}_{2}\right]$ Asp

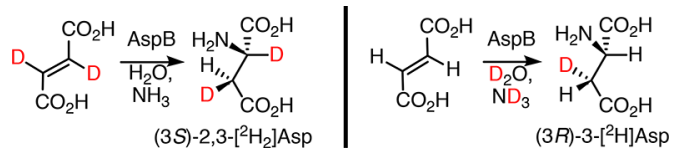

Table 2. Analysis of Isotopic Compositions of the NFL Products from Cultures Fed with Stereospecifically Deuterium-Labeled Aspartic Acids

\begin{tabular}{lccc} 
Asp isotopolog & $\begin{array}{c}\text { amu } \\
\text { shift }\end{array}$ & $\begin{array}{c}\text { \% enrichment of NFL (mean } \\
\pm \text { SD) }\end{array}$ & $\begin{array}{c}p \text { value vs } \\
\text { control }\end{array}$ \\
$(3 R)-3-\left[{ }^{2} \mathrm{H}\right]$ & +1 & $4.1 \pm 0.9$ & 0.0029 \\
$(3 S)-2,3-\left[{ }^{2} \mathrm{H}_{2}\right]$ & +1 & $1.1 \pm 0.4$ & 0.9137 \\
$(3 S)-2,3-\left[{ }^{2} \mathrm{H}_{2}\right]$ & +2 & $0.2 \pm 0.3$ & 0.8139 \\
\hline
\end{tabular}

\section{CONCLUSIONS}

In summary, we have shown that LolO, an Fe/2OG oxygenase, is solely responsible for introducing both $\mathrm{C}-\mathrm{O}$ bonds of the ether bridge of the loline alkaloids. LolO employs a ferryl complex to abstract the endo $\mathrm{H}$ atom from $\mathrm{C} 2$ of AcAP, adds $\mathrm{HO}^{\circ}$ to the nascent radical with retention to give 2-endo- $\mathrm{OH}$ AcAP, abstracts the endo $\mathrm{H}$ atom from $\mathrm{C} 7$ (presumably with a second ferryl complex), and then couples the $\mathrm{C} 2-\mathrm{O}$ atom to the $\mathrm{C} 7$ radical with retention, giving NANL. These findings imply that the bicyclic AcAP substrate binds within LolO so that it effectively cradles the iron cofactor in its concave face, directing both endo hydrogens for abstraction from sequentially formed ferryl intermediates. Whether the geometries of the ferryl intermediates are identical in the two steps and precisely how the fate of the substrate radical is switched from coupling with the $\mathrm{Fe}^{\mathrm{III}}$-hydroxo ligand in the first step to coupling with the C2 alkoxyl group in the second step remain to be established by future structural and mechanistic studies.

\section{MATERIALS AND METHODS}

Overexpression of LolO in E. coli. A gene encoding the same amino acid sequence as the 10102 gene from Ep. uncinata e167 (GenBank accession no. AY723750.1) ${ }^{10}$ but codonoptimized for expression in Escherichia coli was synthesized and inserted into pET28a between the NdeI and BamHI restriction sites by GeneArt (Regensburg, Germany). This construction puts lolO2 under control of the T7 promoter and appends an $\mathrm{N}$-terminal $\mathrm{His}_{6}$ metal ion affinity tag to the protein. The plasmid was used to transform BL21 (DE3) cells (Invitrogen, Carlsbad, CA) for protein expression. The cells were grown at $37{ }^{\circ} \mathrm{C}$ in Luria-Bertani (LB) medium supplemented with 50 $\mu \mathrm{g} / \mathrm{mL}$ kanamycin until an $\mathrm{OD}_{600}$ of $\sim 0.7$ was reached and then cooled to $16{ }^{\circ} \mathrm{C}$. Expression was induced by addition of isopropyl $\beta$-D-1-thiogalactopyranoside to a final concentration of $0.5 \mathrm{mM}$, and cells were shaken overnight at $16{ }^{\circ} \mathrm{C}$ before being harvested by centrifugation.

Purification of Recombinant LolO. All purification steps were performed at $4{ }^{\circ} \mathrm{C}$. The cell paste was resuspended in 3 volumes of lysis buffer containing $100 \mathrm{mM}$ Tris- $\mathrm{HCl}(\mathrm{pH} 7.5)$ with $5 \mathrm{mM}$ imidazole and $1 \mathrm{mM}$ phenylmethanesulfonyl fluoride. The cells were lysed by being passed through a microfluidizer (M110EH-30, Microfluidics, Newton, MA) at $\sim 20,000$ psi for 4 min (approximately two cycles of bursts). The cell debris was then removed by centrifugation, and the supernatant was loaded onto a Ni-NTA agarose column (approximately one-third the volume of the cleared lysate). The column was washed twice with 3 column volumes of the same buffer, and the protein was then eluted by application of $100 \mathrm{mM}$ Tris- $\mathrm{HCl}(\mathrm{pH} 7.5)$ containing $250 \mathrm{mM}$ imidazole. Samples from each step were analyzed by sodium dodecyl sulfate-polyacrylamide gel electrophoresis (SDS-PAGE) visualized by Coomassie blue staining. Eluted fractions containing the target protein were pooled and concentrated with a centrifugal concentrator with a $10 \mathrm{kDa}$ molecular weight cutoff filter (Pall Corp., Port Washington, NY). The concentrated protein was then dialyzed against $50 \mathrm{mM}$ sodium HEPES buffer ( $\mathrm{pH} 7.5$ ) containing $5 \mathrm{mM}$ EDTA to remove iron. Two additional rounds of dialysis with buffer lacking EDTA removed the metal chelator. The enzyme, assessed by SDS-PAGE with Coomassie blue staining to be $>95 \%$ pure, was quantified by UV absorption at $280 \mathrm{~nm}$ by assuming a molar absorptivity $\left(\varepsilon_{280}\right)$ of $76,235 \mathrm{M}^{-1} \mathrm{~cm}^{-1}$ (http://ca.expasy. org).

LC-MS Analysis of LolO Reactions. Constitution of the enzyme reactions is described in the appropriate figure legends. Each reaction solution was passed through a Nanosep centrifugal filter $(0.2 \mu \mathrm{m}$, Pall Corp.) before being injected onto the LC-MS instrument (Agilent 1200 series LC system coupled to an Agilent 6410 QQQ mass spectrometer). A $2 \mu \mathrm{L}$ aliquot of each sample was injected, and compounds were examined by electrospray ionization MS in positive mode. For chromatography, a Kintex $2.6 \mu \mathrm{m}$ HILIC $(150 \mathrm{~mm} \times 2.1 \mathrm{~mm})$ column (Phenomenex, Inc., Torrance, CA) was used at a flow rate of $0.3 \mathrm{~mL} / \mathrm{min}$ with $50 \%$ acetonitrile in water as mobile phase $\mathrm{A}$ and $95 \%$ acetonitrile as mobile phase $\mathrm{B}$. Both solvents were supplemented with $10 \mathrm{mM} \mathrm{NH} \mathrm{CH}_{3} \mathrm{CO}_{2}$ ( $\mathrm{pH}$ 5.8). The elution program was: $100 \% \mathrm{~B}$ for $2.5 \mathrm{~min}$, from 100 to $50 \% \mathrm{~B}$ from 2.5 to $12 \mathrm{~min}, 50 \%$ B to $22 \mathrm{~min}$, from 50 to $100 \%$ B from 22 to $30 \mathrm{~min}$, and $100 \% \mathrm{~B}$ for $10 \mathrm{~min}$ (to prepare for the next injection). Standards of AcAP and NANL for the LC-MS assay were extracted from seeds of Elymus canadensis infected with Epichlö canadensis e $4815 .^{6}$

Isolation of 2-endo-OH-AcAP. An anoxic solution containing $1.5 \mathrm{mM}$ LolO, $1.5 \mathrm{mM} \mathrm{Fe}^{\mathrm{II}}$ [from $\mathrm{Fe}$ $\left.\left(\mathrm{NH}_{4}\right)_{2}\left(\mathrm{SO}_{4}\right)_{2}\right], 3.0 \mathrm{mM}( \pm)$-AcAP, and $1.2 \mathrm{mM} 2 \mathrm{OG}$ (conditions determined empirically to accumulate the greatest quantity of the hydroxylated intermediate) in $50 \mathrm{mM}$ sodium HEPES buffer ( $\mathrm{pH} 7.5$ ) was mixed with an equal volume of cold, $\mathrm{O}_{2}$-saturated buffer. The reaction was allowed to proceed 
for $5 \mathrm{~min}$ at $4{ }^{\circ} \mathrm{C}$, and the mixture was then passed through a centrifugal concentrator with a $10 \mathrm{kDa}$ molecular weight cutoff filter. The flow-through was pooled and lyophilized. This sample was dissolved in a small volume of water and fractionated by preparative HPLC using a Kintex $5 \mu \mathrm{m}$ HILIC $(150 \mathrm{~mm} \times 21.2 \mathrm{~mm})$ column (Phenomenex, Inc.) at a flow rate of $40 \mathrm{~mL} / \mathrm{min}$ with the same mobile phase and elution method as described above. Fractions were collected every $0.2 \mathrm{~min}$, and their composition was determined by LCMS with a reverse-phase Agilent Extend-C18 column (4.6 mm $\times 50 \mathrm{~mm}, 1.8 \mu \mathrm{m}$ particle size). The $\mathrm{C} 18$ column was eluted isocratically with $0.1 \%$ formic acid in water at a flow rate of 0.3 $\mathrm{mL} / \mathrm{min}$. Compounds were examined by electrospray ionization MS in positive mode. The fractions with $>95 \%$ OH-AcAP were pooled and dried by $\mathrm{N}_{2}$ gas, yielding a transparent solid that was used for HRMS and NMR analyses.

Stopped-Flow Absorption and Freeze-Quench Mössbauer Experiments. These experiments were performed essentially as previously described. ${ }^{34}$ Stopped-flow experiments were performed at $5{ }^{\circ} \mathrm{C}$ with an Applied Photophysics Ltd. (Leatherhead, U.K.) SX200 stopped-flow spectrophotometer installed in an anoxic chamber (MBraun, Peabody, MA). The instrument was configured for single mixing with an optical path length of $1 \mathrm{~cm}$. The ferryl absorption feature was acquired by using monochromatic $320 \mathrm{~nm}$ light and a photomultiplier tube (PMT) detector. The $\Delta A_{320}$-versus-time traces were simulated according to a kinetic model with two irreversible steps by using the KinTek Explorer software package (KinTek Corp., Snow Shoe, PA). Fits to the data for the different substrate isotopologs were optimal when the difference in molar absorptivity between the reactant (or product) complex and the ferryl intermediate was taken to be $2000 \mathrm{M}^{-1} \mathrm{~cm}^{-1}$. The error estimates for the kinetic constants were obtained by comparing results of two independent experiments, each with at least three replicates.

To prepare the FQ samples, an $\mathrm{O}_{2}$-free solution containing $2.3 \mathrm{mM}$ LolO, $1.8 \mathrm{mM}{ }^{57} \mathrm{Fe}^{\mathrm{II}}, 12 \mathrm{mM} 2 \mathrm{OG}$, and $3.6 \mathrm{mM}$ ( \pm -AcAP or $2,2,8-\left[{ }^{2} \mathrm{H}_{3}\right]$ AcAP in $50 \mathrm{mM}$ sodium HEPES buffer ( $\mathrm{pH} 7.5$ ) with $20 \%$ glycerol was rapidly mixed with an equal volume of the same buffer saturated with $\mathrm{O}_{2}(1.8 \mathrm{mM}$ $\mathrm{O}_{2}$ ). After being passed through an aging hose of a length appropriate to give the desired reaction time, the solution was rapidly frozen by being injected into cold $\left(-150{ }^{\circ} \mathrm{C}\right) 2$ methylbutane cryosolvent. Packed samples were stored in liquid $\mathrm{N}_{2}$ until they were analyzed. Mössbauer spectra were measured on a spectrometer from WEB Research (Edina, MN) equipped with a SVT-400 cryostat (Janis, Wilmington, MA) to maintain the temperature at $4.2 \mathrm{~K}$. The isomer shifts quoted are relative to the centroid of the spectrum of a metallic foil of $\alpha$-Fe at room temperature. Simulations of the Mössbauer spectra were performed with the WMOSS spectral analysis software package (www.wmoss.org, WEB Research).

\section{ASSOCIATED CONTENT}

\section{S Supporting Information}

The Supporting Information is available free of charge on the ACS Publications website at DOI: 10.1021/acs.biochem.8b00157.

Raw data of LC-MS analysis of LolO products formed with ${ }^{16} \mathrm{O}_{2}$ and ${ }^{18} \mathrm{O}_{2}$, NMR spectra of isolated 2-OHAcAP, assessment of the impact of substrate concentration on ferryl accumulation, comparison of the kinetics from stopped-flow absorption and freeze-quench Mössbauer experiments, protein sequence alignment of LolO with $\mathrm{T} 7 \mathrm{H}$ and the active site of $\mathrm{T} 7 \mathrm{H}$, preparation of the $\mathrm{LolO}$ expression vector, procedure for feeding experiments, explanation of how the biosynthetic pathway from Asp to AcAP impacts determination of the stereochemical course of $\mathrm{C} 2$ hydroxylation, detailed procedures for chemical syntheses, and characterization of the synthetic compounds (PDF)

\section{AUTHOR INFORMATION}

\section{Corresponding Authors}

*E-mail: chris.schardl@uky.edu (for queries regarding natural product biosynthesis).

*E-mail: robert.grossman@uky.edu (for queries regarding synthetic chemistry).

*E-mail: ckrebs@psu.edu (for queries regarding enzymology).

*E-mail: jmb21@psu.edu (for queries regarding enzymology).

ORCID *

Juan Pan: 0000-0003-3307-958X

Wei-chen Chang: 0000-0002-2341-9846

Carsten Krebs: 0000-0002-3302-7053

Robert B. Grossman: 0000-0001-8866-3754

\section{Author Contributions}

${ }^{\perp}$ J.P. and M.B. contributed equally to this work.

\section{Funding}

Supported by National Institutes of Health Grant GM113106 and the U.S. Department of Agriculture (Special Cooperative Agreement 2016-02050844).

\section{Notes}

The authors declare no competing financial interest.

\section{ACKNOWLEDGMENTS}

M.B. and R.B.G. thank Dr. M. Ötzen (University of Groningen, Groningen, The Netherlands) for the AspB expression plasmid, Dr. Connie Wood (University of Kentucky) for help with the statistical analysis of the Asp feeding results, Catherine Denning and Dr. Edith C. Glazer for technical assistance, Dr. AnneFrances Miller for assistance with the NMR experiments, and Dr. Padmaja Nagabhyru for help with feeding experiments. J.P. thanks Dr. Xin Zhang for use of equipment and Dr. Shengbin Zhou for technical assistance. Both M.B. and J.P. thank John May at the University of Kentucky ERTL facility for GC-MS analysis.

\section{REFERENCES}

(1) Robertson, J., and Stevens, K. (2017) Pyrrolizidine alkaloids: occurrence, biology, and chemical synthesis. Nat. Prod. Rep. 34, 62-89.

(2) Bush, L. P., Wilkinson, H. H., and Schardl, C. L. (1997) Bioprotective alkaloids of grass-fungal endophyte symbioses. Plant Physiol. 114, 1-7.

(3) Schardl, C. L., Grossman, R. B., Nagabhyru, P., Faulkner, J. R., and Mallik, U. P. (2007) Loline alkaloids: currencies of mutualism. Phytochemistry 68, 980-996.

(4) Petroski, R. J., and Stanley, D. W. (2009) Natural compounds for pest and weed control. J. Agric. Food Chem. 57, 8171-8179.

(5) Chen, T., Mei, N., and Fu, P. P. (2010) Genotoxicity of pyrrolizidine alkaloids. J. Appl. Toxicol. 30, 183-196.

(6) Pan, J., Bhardwaj, M., Faulkner, J. R., Nagabhyru, P., Charlton, N. D., Higashi, R. M., Miller, A. F., Young, C. A., Grossman, R. B., and Schardl, C. L. (2014) Ether bridge formation in loline alkaloid biosynthesis. Phytochemistry 98, 60-68. 
(7) Pan, J., Bhardwaj, M., Nagabhyru, P., Grossman, R. B., and Schardl, C. L. (2014) Enzymes from fungal and plant origin required for chemical diversification of insecticidal loline alkaloids in grassEpichloe symbiota. PLoS One 9, e115590.

(8) Blankenship, J. D., Houseknecht, J. B., Pal, S., Bush, L. P., Grossman, R. B., and Schardl, C. L. (2005) Biosynthetic precursors of fungal pyrrolizidines, the loline alkaloids. ChemBioChem 6, 10161022.

(9) Spiering, M. J., Wilkinson, H. H., Blankenship, J. D., and Schardl, C. L. (2002) Expressed sequence tags and genes associated with loline alkaloid expression by the fungal endophyte Neotyphodium uncinatum. Fungal Genet. Biol. 36, 242-254.

(10) Spiering, M. J., Moon, C. D., Wilkinson, H. H., and Schardl, C. L. (2005) Gene clusters for insecticidal loline alkaloids in the grassendophytic fungus Neotyphodium uncinatum. Genetics 169, 1403-1414.

(11) Faulkner, J. R., Hussaini, S. R, Blankenship, J. D., Pal, S., Branan, B. M., Grossman, R. B., and Schardl, C. L. (2006) On the sequence of bond formation in loline alkaloid biosynthesis. ChemBioChem 7, 1078-1088.

(12) Spiering, M. J., Faulkner, J. R., Zhang, D. X., Machado, C., Grossman, R. B., and Schardl, C. L. (2008) Role of the LolP cytochrome $\mathrm{P} 450$ monooxygenase in loline alkaloid biosynthesis. Fungal Genet. Biol. 45, 1307-1314.

(13) Hausinger, R. P. (2004) $\mathrm{Fe}(\mathrm{II}) / \alpha$-ketoglutarate-dependent hydroxylases and related enzymes. Crit. Rev. Biochem. Mol. Biol. 39, $21-68$.

(14) Bollinger, J. M., Jr., Chang, W.-c., Matthews, M. L., Martinie, R. J., Boal, A. K., and Krebs, C. (2015) in 2-Oxoglutarate-Dependent Oxygenases (Hausinger, R. P., and Schofield, C. J., Eds.) Royal Society of Chemistry, London.

(15) Hashimoto, T., Kohno, J., and Yamada, Y. (1987) Epoxidation in vivo of hyoscyamine to scopolamine does not involve a dehydration step. Plant Physiol. 84, 144-147.

(16) Li, J., van Belkum, M. J., and Vederas, J. C. (2012) Functional characterization of recombinant hyoscyamine $6 \beta$-hydroxylase from Atropa belladonna. Bioorg. Med. Chem. 20, 4356-4363.

(17) Salowe, S. P., Marsh, E. N. G., and Townsend, C. A. (1990) Purification and characterization of clavaminate synthase from Streptomyces clavuligerus: an unusual oxidative enzyme in natural product biosynthesis. Biochemistry 29, 6499-6508.

(18) Salowe, S. P., Krol, W. J., Iwata-Reuyl, D., and Townsend, C. A. (1991) Elucidation of the order of oxidations and identification of an intermediate in the multistep clavaminate synthase reaction. Biochemistry 30, 2281-2292.

(19) Busby, R. W., Chang, M. D., Busby, R. C., Wimp, J., and Townsend, C. A. (1995) Expression and purification of two isozymes of clavaminate synthase and initial characterization of the iron binding site. General error analysis in polymerase chain reaction amplification. J. Biol. Chem. 270, 4262-4269.

(20) Baggaley, K. H., Brown, A. G., and Schofield, C. J. (1997) Chemistry and biosynthesis of clavulanic acid and other clavams. Nat. Prod. Rep. 14, 309-333.

(21) Pan, J. (2014) Ether bridge formation and chemical diversification in loline alkaloid biosynthesis. Ph.D. Dissertation, University of Kentucky, Lexington, KY.

(22) Tufariello, J. J., Meckler, H., and Winzenberg, K. (1986) Synthesis of the lolium alkaloids. J. Org. Chem. 51, 3556.

(23) Blakemore, P. R., Schulze, V. K., and White, J. D. (2000) Asymmetric synthesis of (+)-loline. Chem. Commun., 1263.

(24) Blakemore, P. R., Kim, S.-K., Schulze, V. K., White, J. D., and Yokochi, A. F. T. (2001) Asymmetric synthesis of (+)-loline, a pyrrolizidine alkaloid from rye grass and tall fescue. J. Chem. Soc., Perkin Trans. 1, 1831-1847.

(25) Cakmak, M., Mayer, P., and Trauner, D. (2011) An efficient synthesis of loline alkaloids. Nat. Chem. 3, 543-545.

(26) Hovey, M. T., Eklund, E. J., Pike, R. D., Mainkar, A. A., and Scheerer, J. R. (2011) Synthesis of ( \pm )-acetylnorloline via stereoselective tethered aminohydroxylation. Org. Lett. 13, 1246-1249.
(27) Miller, K. E., Wright, A. J., Olesen, M. K., Hovey, M. T., and Scheerer, J. R. (2015) Stereoselective synthesis of (+)-loline alkaloid skeleton. J. Org. Chem. 80, 1569-1576.

(28) Ye, J.-L., Liu, Y., Yang, Z.-P., and Huang, P.-Q. (2016) The asymmetric total synthesis of $(+)-\mathrm{N}$-acetyl norloline. Chem. Commun. $52,561-563$.

(29) Chang, W.-c., Guo, Y., Wang, C., Butch, S. E., Rosenzweig, A. C., Boal, A. K., Krebs, C., and Bollinger, J. M., Jr. (2014) Mechanism of the C5 stereoinversion reaction in the biosynthesis of carbapenem antibiotics. Science 343, 1140-1144.

(30) Yan, W., Song, H., Song, F., Guo, Y., Wu, C. H., Sae Her, A., Pu, Y., Wang, S., Naowarojna, N., Weitz, A., Hendrich, M. P., Costello, C. E., Zhang, L., Liu, P., and Zhang, Y. J. (2015) Endoperoxide formation by an $\alpha$-ketoglutarate-dependent mononuclear non-haem iron enzyme. Nature 527, 539-543.

(31) Bollinger, J. M., Jr., and Krebs, C. (2006) Stalking intermediates in oxygen activation by iron enzymes: motivation and method. J. Inorg. Biochem. 100, 586-605.

(32) Krebs, C., Galonić Fujimori, D., Walsh, C. T., and Bollinger, J. M., Jr. (2007) Non-heme Fe(IV)-oxo intermediates. Acc. Chem. Res. $40,484-492$.

(33) Kal, S., and Que, L., Jr. (2017) Dioxygen activation by nonheme iron enzymes with the 2-His-1-carboxylate facial triad that generate high-valent oxoiron oxidants. J. Biol. Inorg. Chem. 22, 339-365.

(34) Price, J. C., Barr, E. W., Tirupati, B., Bollinger, J. M., Jr., and Krebs, C. (2003) The first direct characterization of a high-valent iron intermediate in the reaction of an $\alpha$-ketoglutarate-dependent dioxygenase: a high-spin $\mathrm{Fe}(\mathrm{IV})$ complex in taurine $/ \alpha$-Ketoglutarate dioxygenase (TauD) from Escherichia coli. Biochemistry 42, 74977508.

(35) Hoffart, L. M., Barr, E. W., Guyer, R. B., Bollinger, J. M., Jr., and Krebs, C. (2006) Direct spectroscopic detection of a C-H-cleaving high-spin Fe(IV) complex in a prolyl-4-hydroxylase. Proc. Natl. Acad. Sci. U. S. A. 103, 14738-14743.

(36) Barraclough, P., Dieterich, P., Spray, C. A., and Young, D. W. (2006) Two separate and distinct syntheses of stereospecifically deuteriated samples of (2S)-proline. Org. Biomol. Chem. 4, 1483-1491.

(37) Ooi, T., Miki, T., and Maruoka, K. (2005) Asymmetric synthesis of functionalized aza-cyclic amino acids with quaternary stereocenters by a phase-transfer-catalyzed alkylation strategy. Org. Lett. 7, 191-183.

(38) Back, T. G., Birss, V. I., Edwards, M., and Krishna, M. V. (1988) Study of the reductive cleavage of selenides with nickel boride. A convenient deselenization procedure. J. Org. Chem. 53, 3815-3822.

(39) Matthews, M. L., Krest, C. M., Barr, E. W., Vaillancourt, F. H., Walsh, C. T., Green, M. T., Krebs, C., and Bollinger, J. M., Jr. (2009) Substrate-triggered formation and remarkable stability of the C-H bond-cleaving chloroferryl intermediate in the aliphatic halogenase, SyrB2. Biochemistry 48, 4331-4343.

(40) Galonić, D. P., Barr, E. W., Walsh, C. T., Bollinger, J. M., Jr., and Krebs, C. (2007) Two interconverting $\mathrm{Fe}(\mathrm{IV})$ intermediates in aliphatic chlorination by the halogenase CytC3. Nat. Chem. Biol. 3, 113-116.

(41) McCusker, K. P., and Klinman, J. P. (2009) Modular behavior of TauD provides insight into the origin of specificity in $\alpha$-ketoglutaratedependent nonheme iron oxygenases. Proc. Natl. Acad. Sci. U. S. A. 106, 19791-19795.

(42) Tamanaha, E., Zhang, B., Guo, Y., Chang, W.-c., Barr, E. W. Xing, G., St. Clair, J., Ye, S., Neese, F., Bollinger, J. M., Jr., and Krebs, C. (2016) Spectroscopic evidence for the two C-H-cleaving intermediates of Aspergillus nidulans isopenicillin $N$ synthase. J. Am. Chem. Soc. 138, 8862-8874.

(43) Baldwin, J. E., and Bradley, M. (1990) Isopenicillin $N$ synthase: mechanistic studies. Chem. Rev. 90, 1079-1088.

(44) Chang, W.-c., Dey, M., Liu, P. H., Mansoorabadi, S. O., Moon, S.-j., Zhao, Z. K., Drennan, C. L., and Liu, H. W. (2013) Mechanistic studies of an unprecedented enzyme-catalysed 1,2-phosphonomigration reaction. Nature 496, 114-118.

(45) Wang, C., Chang, W.-c., Guo, Y., Huang, H., Peck, S. C., Pandelia, M.-E., Lin, G.-m., Liu, H.-w., Krebs, C., and Bollinger, J. M. 
(2013) Evidence that the Fosfomycin-Producing Epoxidase, HppE, Is a Non-Heme-Iron Peroxidase. Science 342, 991-995.

(46) Petroski, R. J., Yates, S. G., Weisleder, D., and Powell, R. G. (1989) Isolation, semi-synthesis, and NMR spectral studies of loline alkaloids. J. Nat. Prod. 52, 810-817.

(47) Weiner, B., Poelarends, G. J., Janssen, D. B., and Feringa, B. L. (2008) Biocatalytic enantioselective synthesis of $\mathrm{N}$-substituted aspartic acids by aspartate ammonia lyase. Chem. - Eur. J. 14, 10094-10100.

(48) Gani, D., and Young, D. W. (1983) Synthesis of L-serine stereospecifically labelled at C-3 with deuterium. J. Chem. Soc., Perkin Trans. 1, 2393-2398.

(49) Field, S. J., and Young, D. W. (1983) Synthesis of L-glutamic acid labelled stereospecifically at C-3 with deuterium and nonstereospecifically at C-4 with tritium. J. Chem. Soc., Perkin Trans. 1, 2387-2392.

(50) Barclay, F., Chrystal, E., and Gani, D. (1996) Synthesis of (2S)O-phosphohomoserine and its C-2 deuteriated and C-3 chirally deuteriated isotopomers: probes for the pyridoxal phosphate-dependent threonine synthase reaction. J. Chem. Soc., Perkin Trans. 1, 683689. 